\title{
Probiotics as a therapeutic strategy in obesity and overweight: a systematic review
}

X.M. Tomé-Castro ${ }^{1}$, M. Rodriguez-Arrastia ${ }^{2,3, *\left({ }^{*} \text { (orresponding author) }\right.}$, D. Cardona ${ }^{1,4, *(\text { corresponding }}$ author) , L. Rueda-Ruzafa ${ }^{5}$, G. Molina-Torres ${ }^{6}$, P. Roman ${ }^{1,4,5}$

${ }^{l}$ Faculty of Health Sciences, Department of Nursing Science, Physiotherapy and Medicine, University of Almeria, Almeria (Spain); ${ }^{2}$ Faculty of Health Sciences, PreDepartment of Nursing, Jaume I University, Castello de la Plana (Spain); ${ }^{3}$ Research Group CYS, Faculty of Health Sciences, Jaume I University, Castello de la Plana (Spain); ${ }^{4}$ Health Research Centre, University of Almeria, Almeria (Spain); ${ }^{5}$ Research Group CTS451 Health Sciences, University of Almeria, Almeria (Spain); ${ }^{6}$ Faculty of Health Sciences, Department of Physiotherapy, University of Granada, Melilla (Spain); arrastia@uji.es; dcardona@ual.es

\begin{abstract}
Obesity and overweight are two of the most health challenges with an increasing prevalence in recent years, in which several complications have been identified to have a high impact in patients' health conditions. In this vein, an increasing interest in the gut microbiota has emerged as a target for therapeutic strategies in obesity and overweight due to its direct relation with the aforementioned health conditions and complications. Thus, the aim of this study was to evaluate the efficacy of probiotics as a therapeutic strategy in the management of obesity and overweight. A systematic review of randomized controlled trials was carried out in 6 databases until May 2019 to assess the use of probiotics in obesity and overweight patients. The Jadad Scale was used to assess the quality of clinical trials. Twenty-three clinical trials published between 2000 and 2019 met the inclusion criteria. The role of probiotics in reducing body mass index and weight as well as changing the visceral abdominal fat area, waist and hip circumference were shown in 14 of 23 trials $(60.87 \%)$; 14 trials $(60.87 \%)$ showed changes on patients' fatty acids and biomarkers; and 4 trials $(17.39 \%)$ studied the role of the gut microbiota in obese and overweight patients. Some probiotics strains are shown to be effective in reducing body mass index and hip circumference. This review provides evidence of successful results in weight loss using probiotic groups.
\end{abstract}

Keywords: gut microbiota, obesity, overweight, probiotic 


\section{Introduction}

Two of the most important health challenges worldwide are obesity and overweight, which also constitute a global risk for mortality. In this matter, 1.9 billion adults were overweight in 2016, of whom 650 million were obese (Bentham et al., 2017; Borgeraas et al., 2018; Hadi et al., 2018; WHO, 2018). Moreover, obesity and overweight have been shown to have several medical complications such as cardiovascular diseases, metabolic disorders, respiratory disorders, muscle-skeletal disorders and social and psychological alterations, among others and therefore, the increasing interest for their prevention and treatment acquire great importance between health professionals (Hadi et al., 2018; Hampl and Campbell, 2015; King and Ajjan, 2016; Rizzetto et al., 2018; Wang et al., 2019).

In recent years, the gut microbiota has been widely studied in nutrition for its implication in the pathophysiology for obesity and overweight (Gérard, 2016; Gomes et al., 2018; Maruvada et al., 2017; Turnbaugh et al., 2006). This implication has been shown to be related to its ability to modulate gastrointestinal (GI) functions such as intestinal motility (Fayfman et al., 2019) and permeability (Karl et al., 2017), mucosal immune function (Rizzetto et al., 2018), and activity in the enteric nervous system (Heiss and Olofsson, 2019; Obata and Pachnis, 2016). Obesity is widely associated with a lower bacterial diversity and an increased Firmicutes/Bacteroidetes ratio, an event known as intestinal dysbiosis (Gomes et al., 2018). It is worth noting that both, a recent systematic review (Crovesy et al., 2020) and a meta-analysis (Mitev and Taleski, 2019), corroborate this imbalance Firmicutes/Bacteroidetes ratio in obese individuals, pointing to a different microbiota profile for individuals with obesity, which decreases if the person loses weight.

In this context, intestinal bacteria species are key in the synthesis of short-chain fatty acids (SCFA) by decreasing inflammation (Zhai et al., 2019), intervening in the degradation of complex carbohydrates (Flint et al., 2012) and transforming primary bile acids into secondary ones, which are fundamental for the emulsification, degradation and absorption of cholesterol, fats and fat-soluble vitamins (Ramírez-Pérez et al., 2017).

The gut microbiota can be modulated by different ways, such as diet, faecal microbial transplantation (de Groot et al., 2017; Paramsothy et al., 2017), drugs, especially antibiotics (Becattini et al., 2016; Ianiro et al., 2016; Noh et al., 2017), as well as probiotics, prebiotics and symbiotics (Ferrarese et al., 2018). In this sense, probiotics have been defined as living organisms that consumed in adequate amounts provide beneficial health effects (FAO/WHO, 2001). These probiotics and other gut microbiota modulators may alter the secretion of hormones, neurotransmitters, and inflammatory factors, and therefore preventing food intake triggers that lead to weight gain (Aoun et al., 2020). Experimental studies have shown anti-obesogenic effects after probiotics treatments, mainly Lactobacillus and Bifidobacterium, characterized by a lower weight gain as well as a reduction on fat accumulation (Ejtahed et al., 2019). Some of the mechanisms of action proposed include induction in fatty acid oxidation genes or regulation of the genes involved in lipid metabolism (Dahiya et al., 2017). In addition, some strains of Lactobacilli can produce bacteriocins, antimicrobial peptides that are related with body weight. Moreover, probiotics prevent dysbiosis and protect the intestinal barrier through the modulation of cytoskeletal (Cerdó et al., 2019). Another 
mechanism of action postulated is related to their ability to compete with microorganisms for binding sites by antagonizing these pathogens or by modulating the recipients' immune response. There are several microorganisms recognized as probiotics and not all of them have the same effects and mechanisms of action, and thereby further discussion using probiotics as a therapeutic strategy is needed (Hadi et al., 2018; Sanchis-Chordà et al., 2018; Sarao and Arora, 2017). Thus, the aim of this study was to evaluate the efficacy of probiotics as a therapeutic strategy in the management of obesity and overweight in adults, and their effect in other biomarkers as secondary outcomes.

\section{Methods}

\section{Search strategy}

In May 2019, a systematic review of clinical trials was conducted. The review process followed the Preferred Reporting Items for Systematic Reviews and Meta-Analyzes (PRISMA) statement (Moher et al., 2009). A structured Patient-Intervention-Outcomes (PIO) question was planned as follows (Stone, 2002): Does probiotics constitute an effective therapeutic strategy in obese or overweight patients?

This study was carried out in main health science databases: PubMed, Cochrane Library, ProQuest, CINAHL, Science Direct and Scopus; combining natural language and structured language, using the following MeSH terms: "probiotics", "microbiota", "obesity" and "overweight". The search strategy used was adapted to each database (see Supplement File 1). The only filter used was "clinical trials" as article type in PubMed.

\section{Selection criteria}

The following inclusion criteria were used: (i) randomized clinical trials, (ii) papers related to the aim of the study; evaluate the efficacy of probiotics as a therapeutic strategy in the management of loss weight, obesity and/or overweight, (iii) adults aged 18-65 years, (iv) language of publication in English, (iv) included at least one of the following variables: BMI and change in weight, and (v) studies with at least a probiotic intervention. The exclusion criteria were: (i) studies conducted in patients with HIV, cancer, diabetes, cardiovascular diseases, pregnant women, and breastfeeding, (ii) re-publications, and (iii) studies within animals.

\section{Screening of studies}

The eligibility of studies was done in three phases. The first phase consisted in reading the title of articles identified throughout the database searching. Once selected, all abstracts were reviewed in a second phase. And finally, full-text reading was used when needed for clarification and in order to discern the suitability of selecting the paper for its analysis. The eligibility process was done by the two first authors (MRA, XM) independently and in duplicate, if consensus could not be achieved, a third author (PR) was consulted. In relation to the included studies, a bibliometric analysis was carried out 
on the following variables: (i) methods, (ii) participants, (iii) criteria of the studies, (iv) duration of the intervention, (v) probiotics strains used and (vi) results.

\section{Quality of evidence}

Besides, a critical reading analysis was performed with the Jadad Scale of Clinical Trials to assess the methodological quality of the analysed studies. This is a scale with five simple items and it has known reliability and external validity. A score below 3 points indicates low quality based on the quality of randomization, double blinding, and dropouts extracted of each study (Jadad et al., 1996). Any discrepancy was discussed by authors in order to reach a consensus and preserve the reliability of the findings.

\section{Results}

\section{Study selection}

Combining the aforementioned terms resulted in 586 articles [PubMed $(n=105)$, Cochrane Library $(n=154)$, Proquest $(n=97)$, CINAHL $(n=98)$, Science Direct $(n=62)$, and Scopus $(n=70)]$, from which 345 were discarded by duplicity. From the 241 remaining documents, 218 were excluded according to our selection criteria. And finally, a total of 23 documents were included in this systematic review for their analysis. The review and selection processes are detailed in Figure 1.

\section{[INSERT FIGURE 1 AROUND HERE]}

\section{Literature characteristics of included studies}

Trials and patients' characteristics are compiled in Table 1. Overall, participants in most of the 23 analysed articles were obese and overweight volunteers, except for five studies (Bernini et al., 2016; Hulston et al., 2015; Leber et al., 2012; Ogawa et al., 2014; Osterberg et al., 2015). In three trials (Bernini et al., 2016; Chung et al., 2016; Zarrati et al., 2013), the gender of participants was not specified and only women participated in Lee and collaborators' (2014) and Madjd and collaborators' trials (2016), as well as only men participated in the study of Osterberg and collaborators' (2015). Inclusion criteria involved an age between 18 and 75 years old (mean age 44.1 years old), enrolling a total of 1996 patients. All manuscripts were published between 2000 and 2019, and 12 clinical trials $(52.17 \%)$ were not registered in a clinical trial registry. Most of the studies were performed in Asia $(n=13)$, Europe $(n=6)$, America $(n=3)$ and only one was from Oceania $(n=1) .12$ studies $(52.17 \%)$ included participants who consumed a single strain of probiotics, and 11 studies (47.83\%) included participants who consumed two or multiple strains of probiotics. Also, probiotics were administered in different forms and duration of the probiotic supplementation ranged from 4 weeks to 6 months. One study evaluated the effects of probiotic in two different presentations (Ivey et al., 2014). The range dose was between $10^{6}$ from $10^{12} \mathrm{CFU} /$ day, although in two studies the dose in CFU was not 
indicated (Hulston et al., 2015; Nakamura et al., 2016). Doses of the same probiotic are evaluated in two studies (Kadooka et al., 2013; Kim et al., 2018) and other two studies compared the effect between heat-killed or living strain (Higashikawa et al., 2016; Pedret et al., 2019). Nevertheless, it is noteworthy that two of the studies analysed evaluate also the effect of prebiotic and symbiotic (Hibberd et al., 2019; Stenman et al., 2016). Moreover, microbial composition changes were reported as outcomes only in 3 studies (13.04\%). And finally, 16 studies (69.57\%) reported positive results on different parameters (e.g. body weight, fat mass, BMI, biomarkers, etc.), whilst 7 studies (30.43\%) reported no impact in their results.

\section{[INSERT TABLE 1 AROUND HERE]}

\section{Effects of probiotics in weight and BMI}

The role of probiotics in reducing BMI and weight or changing the visceral abdominal fat area, waist and hip circumference was demonstrated in 12 of 23 trials (52.17\%) (Bernini et al., 2016; Chung et al., 2016; Higashikawa et al., 2016; Kadooka et al., 2010, 2013; Kim et al., 2018; Lee et al., 2014; Nakamura et al., 2016; Osterberg et al., 2015; Pedret et al., 2019; Stenman et al., 2016; Takahashi et al., 2016). It is interesting to note that 9 of these studies were performed in Asia where the population's BMI is lower than in America or Europe (Castaner et al., 2018).

The probiotics strains employed along these studies were: Bifidobacterium animalis subsp. lactis, subsp nov. HN019; Lactobacillus reuteri JBD301, Pediococcus pentosaceus LP28, Lactobacillus gasseri BNR17, Lactobacillus amylovorus CP1563, Bifidobacterium animalis spp. lactis BA8145, Bifidobacterium animalis spp. lactis B420, and Bifidobacterium lactis GCL2505. In other clinical trials, probiotics were used in combination (Streptococcus thermophilus and Lactobacillus delbrueckii ssp. bulgaricus, along with Lactobacillus gasseri LG2055), (Streptococcus thermophiles KCTC 11870BP, Lactobacillus plantarum KCTC 107892BP, Lactobacillus acidophilus KCTC 11906BP, Lactobacillus rhamnosus KCTC 12202BP, Bifidobacterium lactis KCTC 11904BP, Lactobacillus longum KCTC 12200BP, and Bifidobacterium breve KCTC 12201BP), (Streptococcus thermophilus DSM24731, and Lactobacillus acidophilus DSM24735). The treatment period varied from 4 weeks to 6 months, being the 12 -week period the most commonly used. The effects of probiotic over gender were only indicated in the study of Pedret and collaborators (2019), where the probiotic effects were observed only in women.

On the other hand, 7 trials $(30.43 \%)$ indicated the poor efficacy of probiotics in reducing BMI, weight or other anthropometric parameters (Hibberd et al., 2019; Hulston et al., 2015; Ivey et al., 2014; Jung et al., 2013; Leber et al., 2012; Lee et al., 2014; Madjd et al., 2016). Only in 3 studies (13.04\%) the anthropometric measures were not reported as outcomes (Culpepper et al., 2019; Ogawa et al., 2014; Zarrati et al., 2014). The probiotics used in these studies included Lactobacillus gasseri BNR17, Treptococcus thermophiles, Lactobacillus bulgaricus, Bifidobacterium lactis BB, Lactobacillus acidophilus LA, Lactobacillus acidophilus La5, Bifidobacterium animalis subsp lactis BB12, Bifidobacterium subtilis R0179, Streptococcus thermophiles KCTC 11870BP, Lactobacillus plantarum KCTC 107892BP, Lactobacillus acidophilus KCTC 11906BP, 
Lactobacillus rhamnosus KCTC 12202BP, Bifidobacterium lactis KCTC 11904BP, Lactobacillus longum KCTC 12200BP, and Bifidobacterium breve KCTC 12201BP. The treatment of these studies ranges from 4 weeks to 3 months.

\section{Effects of probiotics in other biomarkers}

In reference to the changes on patients' fatty acids and biomarkers, a total of 13 trials (56.52\%) showed changes (Agerholm-Larsen et al., 2000; Bernini et al., 2016; Hibberd et al., 2019; Hulston et al., 2015; Ivey et al., 2014; Kadooka et al., 2013; Madjd et al., 2016; Nakamura et al., 2016; Ogawa et al., 2014; Pedret et al., 2019; Stenman et al., 2016; Zarrati et al., 2013, 2014). In that sense, the literature reviewed showed an impact from probiotics treatments, where the improved metabolic health status was indicated as a decrease of total cholesterol, LDL, triglyceride concentration, and a reduction of esterified fatty acid (OFLT), non-esterified fatty acids (NEFA) and triacylglycerol (TAG). Indeed, there were reductions in total cholesterol, LDL, and an increase in the insulin resistance index (HOMA-IR) according to the study of Madjd and collaborators (2016). Likewise, the HOMA-IR was also reduced in Ivey and collaborators' results (2014) and in Pedret and collaborators' (2019). Nevertheless, it was not reported any reduction in total cholesterol, HDL, or triglycerides. On the other hand, a slight improvement was shown in Madjd and collaborators' (2016) and Culpepper and collaborators' findings (2019), but not significant enough to demonstrate the role of probiotics. Conversely, there were studies which showed negative effects correlated with endotoxin levels and total cholesterol, HDL, LDL and triglycerides, but there were no significant changes in their results (Jung et al., 2013; Lee et al., 2014). In the same manner, inconclusive results for changes in these biomarkers were reported in one study (Ivey et al., 2014).

Conversely, impact on immune systems were also reported in 6 studies (Agerholm-Larsen et al., 2000; Bernini et al., 2016; Stenman et al., 2016; Zarrati et al., 2013, 2014). Five of these trials showed changes in levels of zonulin and CRP, a reduction of leptin levels as well as a stimulation of the IL-10 production and therefore an inhibition of TH1 and its cytokines. Concerning to the impact on the gut microbiota, three studies $(13.04 \%)$ evaluated that relationship between fatty acids reduction and probiotics. Stenman et al. (2016) observed an increase of the concentration of faecal propionic acid, butyric acid and valeric acid, as well as total faecal SCFA concentrations. However, no significant changes in SCFA, amino acids were noticed by Hibberd and collaborators (2019) and Pedret and collaborators (2019). In the same line, four studies (17.39\%) assessed the effects of probiotics treatments over gut microbiota, which found an increase of bacterial composition. In that sense, Pedret and collaborators (2019) identified an increase in Akkermansia spp. after 3 months of treatment with Bifidobacterium animalis spp. lactis. Takahashi and collaborators (2016) observed an increased number of faecal bifidobacterial after 12 weeks of treatment with Bifidobacterium lactis GCL2505. Lee and collaborators (2014) noticed an increase of the levels of B. breve, B. lactis, and L. rhamnosus after the treatment with a probiotic multispecies during 6 weeks. And finally, Osterberg and collaborators (2015) detected greater faecal bacterial enrichment in groups treated with a probiotic multispecies during 4 weeks. 


\section{Quality assessment}

The average quality of the analysed studies scored 3.52 in the Jadad Scale (Table 2). Its variability was ranged from 2 (in four studies), 3 (in eight studies), 4 (in six studies) to 5 (in five studies), and none had an unacceptable reporting quality (lower than 1). As for potential sources of sponsorship bias, 8 analysed studies received funding from different manufacturers (Bernini et al., 2016; Chung et al., 2016; Hibberd et al., 2019; Hulston et al., 2015; Lee et al., 2014; Nakamura et al., 2016; Ogawa et al., 2014; Takahashi et al., 2016).

\section{[INSERT TABLE 2 AROUND HERE]}

\section{Discussion}

The aim of the present study was to evaluate the efficacy of probiotics as a therapeutic strategy against obesity and overweight on humans. In this sense, this systematic review from 23 randomized controlled trials showed the beneficial effects that probiotics may have in obesity and overweight management. That is, a reduction in BMI and weight among other anthropometric measures, which were observed in 12 of 23 trials $(52.17 \%)$; along with 14 trials $(60.87 \%)$ that showed changes on patients' fatty acids and other biomarkers. Our results are according to other found in previous reviews about the topic (Azad et al., 2018; Borgeraas et al., 2018; Rouxinol-Dias et al., 2016; Wang et al., 2019).

There exists a wide variety of strains which can be used in order to obtain these beneficial health effects. Thus, Streptococcus thermophilus strain was the most widely used (Bernini et al., 2016; Kadooka et al., 2013; Lee et al., 2014; Osterberg et al., 2015; Zarrati et al., 2014). Almost half of the reviewed manuscripts employed a multi-strain probiotics (Agerholm-Larsen et al., 2000; Culpepper et al., 2019; Ivey et al., 2014; Kadooka et al., 2010, 2013; Lee et al., 2014; Madjd et al., 2016; Ogawa et al., 2014; Osterberg et al., 2015; Zarrati et al., 2013, 2014), which seems to be more effective than a single strain according to a recent meta-analysis (Koutnikova et al., 2019). Regarding the duration of the intervention, the most common treatment was 12 weeks, that seems to be enough to demonstrate changes in body weight. Others authors, such as Bernini and collaborators (2016), Madjd and collaborators (2016), or Ogawa and collaborators (2014), among others, used Lactobacillus bulgaricus strain with similar significant results in a length of time alike. Many authors (Agerholm-Larsen et al., 2000; Chung et al., 2016; Higashikawa et al., 2016; Kadooka et al., 2010; Kim et al., 2018) agree in the positive effects of probiotics for changing metric parameters like weight and BMI, particularly in the abdominal region, waist and hip circumference, diastolic blood pressure, fat percentage, whole body fat, and visceral fat. In this sense, Pedret and collaborators' results (2019) demonstrated how the consumption of probiotics such as BA8145 improves biomarkers of anthropometric adiposity such as visceral fat area, BMI, waist circumference, waistto-hip ratio and conicity index, particularly in women, and appears to constitute a complementary strategy in obesity.

Regarding to the effects of probiotics in other biomarker measures, Stenman and collaborators' (2016), and Zarrati and collaborators' results (2014) among others revealed how this process induced changes at protein levels like levels of zonulin and CRP, besides 
immunology and metabolic changes. For that matter, Ogawa and collaborators (2014) showed how levels of post-nephric NEFA and levels of TAG in the active fermented milk $(\mathrm{FM})$ period were lower $(\mathrm{p}<0.005)$ than in the FM control group.

New probiotics such as Akkermansia muciniphila have a negative correlation with overweight and obesity (Vallianou et al., 2020). Results from both preclinical and clinical research have showed declined abundance of $A$. muciniphila in obesity and metabolic syndromes (Xu et al., 2020). This probiotic has been effective to improved metabolic biomarkers like insulin sensitivity, total cholesterol and also decrease inflammation (Depommier et al., 2019; Li et al., 2016). The role of $A$. muciniphila in body metabolism revealed to be a great option treatment of metabolic disorders associated with obesity. In fact, A. muciniphila impacts on SCFA production, which can have effects on glucose and lipid homeostasis (Xu et al., 2020).

In this matter, a recent review points out to three mechanism of probiotics action over obesity: antimicrobial activity, immunomodulation and reduction of permeability barrier (Abenavoli et al., 2019). Treatments with a mixture of probiotics have been shown effective to alter host inflammation, adipose tissue hormone levels, and intestinal microbial composition. In that sense, Al-Muzafar and Amin's results (2017) showed that co-administrated with a high fat diet induced changes in intestinal microbiota, which reduced serum lipid profiles and inflammatory biomarkers. Furthermore, some studies indicate that certain bacterial strains are able to decrease the inflammatory environment associated with gastrointestinal dysfunction in animal models of obesity and obese patients, whereas others find changes in levels of certain proteins involved in the energy balance (Park et al., 2019; Sun et al., 2020; Zarrati et al., 2013).

One possible mechanism of action of probiotics over obesity could be their impact on cytokines induction (Maldonado-Galdeano et al., 2019). Thereby, Zarrati and collaborators (2013) showed that probiotics (Lactobacillus acidophilus LA5, Bifidobacterium BB12 and Lactobacillus casei DN001) also stimulate the production of IL-10 and therefore inhibit TH1 and its cytokines in an 8-week period. As these probiotics can also modify lipid and glucidic homeostasis, mRNA levels of peroxisome proliferatoractivated receptor-gamma (PPAR- $\gamma$ ) and PGC- $1 \alpha$, proteins that interact with PPAR- $\gamma$ were significantly elevated, promoting the interaction of both and improving obesity in HFD mice after the administration of L. amylovorus KU4 (Park et al., 2019).

In this sense, treatments with L. plantarum CGMCC1.557, L. fermentum CGMCC1.1880, B. breve CICC 6182 and L.casei CRL 431 showed an improvement in the composition of the intestinal bacteria, along with reduced levels of IL-1 $\beta$, IL-6, IL-17, TNF- $\alpha$ and LPS and increased levels of IL-10, which had anti-inflammatory properties in mice fed with high-fat diets (HFD mice) and E.coli-induced endotoxemia (Novotny-Núñez et al., 2015; Sun et al., 2020). Similarly, a probiotic mixture containing B. animalis VLK and VKB and $L$. casei IMV B-7280 increased the levels of IL-4, IL- 10 and TGF- $\beta$ in rats with monosodium glutamate-induced obesity (Falalyeyeva et al., 2017). In this vein, $L$. plantarum was shown as a promising strain for the reduction of hypercholesterolemia and adipogenesis in vitro experiments (Huang et al., 2019).

In spite of the consistency among the reviewed trials, discrepancies in strain differences and individuals' genotype were found. So, both Hulston and collaborators (2015), Leber 
and collaborators (2012), as well as Madjd and collaborators (2016) showed poor effects of probiotics over anthropometric parameters. In the same line, a recent metanalysis showed that oral probiotics and/or symbiotic administration have no effect on body weight or BMI, although reduced minimally waist circumference (Suzumura et al., 2019). However, there are only two studies that did not find different effects between control and probiotics groups (Jung et al., 2013; Lee et al., 2014). That aside, despite there were no significant changes in body weight (Lee et al., 2014) and total cholesterol, HDL, LDL and triglycerides levels (Jung et al., 2013), an increase in bacteria composition was observed.

It is interesting to note that the form of the probiotic has been also compared in some of the studies reviewed. Ivey and collaborators (2014) found that probiotic yogurt (PY) increased the HOMA-IR and fasting glucose concentration whilst the probiotic capsules did not. Also, heat-killed strain seems to be more effective than live strain (Higashikawa et al., 2016; Pedret et al., 2019).

On the other hand, dietary interventions have been evaluated in two studies (Madjd et al., 2016; Osterberg et al., 2015). Relevant results were obtained from Osterberg and collaborators (2015) who observed that a multispecies probiotic reduced the increase in body weight caused by a high-fat diet. The relationship between gut microbiota, obesity, diet and other factors such as physical exercise has been reviewed (Brahe et al., 2016). In fact, an increase of intestinal bacteria was detected in those patients who experienced weight loss through diet and exercise. As Hadi and collaborators' (2018), and Prados-Bo and collaborators' results (2015) suggest, the effectiveness of lifestyle interventions in weight loss seems to be influenced by the composition of each individual's microbiota. It is well-known that one of the main activities of the intestine bacteria is to break down substrates like dietary fibre. As result of this degradation, SCFA are produced, interplaying between diet, gut microbiota and host energy metabolism. SCFA exert multiple effects on host metabolism by acting on glucose and lipid metabolism in various tissues, notably in liver, adipose tissue and muscle in which they act on insulin signalling and even, with a key role in gut-brain axis (Hadi et al., 2018; Rizzetto et al., 2018).

Regarding potential mechanism to explain the aforementioned effects, there is growing evidence that support a link between the gut microbiota, SCFAs and obesity (Wang et al., 2019). Gut microbiota has been evaluated only in four studies, but all of them found an increase of bacterial diversity or richness or an increased abundance in certain species (Lee et al., 2014; Osterberg et al., 2015; Pedret et al., 2019; Takahashi et al., 2016). This bacterial richness will modify the production of SCFA and consequently will lead to beneficial metabolic impact. In that matter, only Stenman and collaborators' results (2016) showed an increased in the concentration of faecal propionic acid, butyric acid and valeric acid, as well as total faecal SCFA concentrations, whilst no significant changes in SCFA were observed by Hibberd and collaborators (2019) and Pedret and collaborators (2019).

Other systematics reviews and metanalysis have evaluated the effects of probiotics treatments in obesity (Aoun et al., 2020; Borgeraas et al., 2018; Cerdó et al., 2019; Dahiya et al., 2017; Ejtahed et al., 2019; Koutnikova et al., 2019; Suzumura et al., 2019). Their results are according to our systematic review, showing that probiotics consumption improves anthropometric parameters and BMI. However, the exact mechanism of action still needs to be elucidated as it seems to be specific for each probiotic species and strain. 
Nevertheless, more studies are necessary according to López-Moreno and collaborators (2020), due to the high variability between studies and lack of standardized protocols.

\section{Limitations}

Nevertheless, there is a number of limitations to consider when interpreting the results of this review. First, the number of excluded studies has been noteworthy due to its low methodological quality. Indeed, twelve clinical trials were not registered, which may have a risk of reporting bias. Our findings are based on consistent trials, following Jadad Scale. In addition, differences on strains, length of interventions and population of the studies may constitute confounding factors, and thereby generalizations should be made with caution. Due to the limited number of studies included, the effects of probiotics treatments in the prevention and treatment of overweight and obesity still need more consistent work, specially to identify potential mechanisms of action. Lastly, a wider strategy was used, using both probiotic and microbiota as natural and structured terms, to include the use of specific genera as some authors refer to probiotics by the specific genus used in their studies.

In summary, this study contributes to the existing literature proving the efficacy of probiotics as a therapeutic strategy against obesity and overweight on humans. Further research is needed with larger sample groups and length of interventions in order to confirm the beneficial effects of probiotics as a therapeutic strategy.

\section{Conclusions}

In conclusion, our findings showed that some probiotics strains (Streptococcus thermophilus, Lactobacillus bulgaricus, Lactobacillus acidophilus, among others) are a valid therapeutic strategy to use against obesity and overweight by modifying metric parameters and other biomarkers, especially when multi-strains rather than single strains are used. The beneficial variation among different strains has been similar and thereby, all of them may be considered as a possible strategy to regulate the gut microbiota, being a 12-week treatment the most widely used and effective. Furthermore, these probiotics have showed to be more effective in reducing BMI and hip circumference. Also, probiotics administration could produce a beneficial effect in many biomarkers, such as cholesterol, LDL, triglyceride concentration or in HOMA-IR. In this vein, the composition of each individual's microbiota seems to have an influence in avoiding a body weight increase during dietary interventions, where multispecies probiotics have been shown to be effective.

\section{Conflict of interests}

The authors declare no conflict of interests. 
Probiotics in obesity and overweight

\section{Funding}

This research received no specific grant from any funding agency in the public, commercial, or not-for-profit sectors.

\section{References}

Abenavoli, L., Scarpellini, E., Colica, C., Boccuto, L., Salehi, B., Sharifi-Rad, J., Aiello, V., Romano, B., De Lorenzo, A., Izzo, A.A., \& Capasso, R. (2019). Gut Microbiota and Obesity: A Role for Probiotics. Nutrients, 11(11). https://doi.org/10.3390/nu11112690

Agerholm-Larsen, L., Raben, A., Haulrik, N., Hansen, A.S., Manders, M., \& Astrup, A. (2000). Effect of 8-week intake of probiotic milk products on risk factors for cardiovascular diseases. European Journal of Clinical Nutrition, 54(4), 288-297. https://doi.org/10.1038/sj.ejcn.1600937

Al-Muzafar, H.M., \& Amin, K.A. (2017). Probiotic mixture improves fatty liver disease by virtue of its action on lipid profiles, leptin, and inflammatory biomarkers. BMC Complementary and Alternative Medicine, 17(1), 43. https://doi.org/10.1186/s12906-016-1540-z

Aoun, A., Darwish, F., \& Hamod, N. (2020). The Influence of the Gut Microbiome on Obesity in Adults and the Role of Probiotics, Prebiotics, and Synbiotics for Weight Loss. Preventive Nutrition and Food Science, 25(2), 113-123. https://doi.org/10.3746/pnf.2020.25.2.113

Azad, M.A.K., Sarker, M., Li, T., \& Yin, J. (2018). Probiotic Species in the Modulation of Gut Microbiota: An Overview. BioMed Research International, 2018. https://doi.org/10.1155/2018/9478630

Becattini, S., Taur, Y., \& Pamer, E.G. (2016). Antibiotic-Induced Changes in the Intestinal Microbiota and Disease. Trends in Molecular Medicine, 22(6), 458-478. https://doi.org/10.1016/j.molmed.2016.04.003

Bentham, J., Di Cesare, M., Bilano, V., Bixby, H., Zhou, B., Stevens, G.A., Riley, L.M., Taddei, C., Hajifathalian, K., Lu, Y., Savin, S., Cowan, M.J., Paciorek, C.J., Chirita-Emandi, A., Hayes, A.J., Katz, J., Kelishadi, R., Kengne, A. P., Khang, Y. H., ... Cisneros, J.Z. (2017). Worldwide trends in body-mass index, underweight, overweight, and obesity from 1975 to 2016: A pooled analysis of 2416 population-based measurement studies in 128.9 million children, adolescents, and adults. The Lancet, 390(10113), 2627-2642. https://doi.org/10.1016/S01406736(17)32129-3

Bernini, L.J., Simão, A.N.C., Alfieri, D.F., Lozovoy, M.A.B., Mari, N.L., de Souza, C. H.B., Dichi, I., \& Costa, G.N. (2016). Beneficial effects of Bifidobacterium lactis on lipid profile and cytokines in patients with metabolic syndrome: A randomized 
trial. Effects of probiotics on metabolic syndrome. Nutrition, 32(6), 716-719. https://doi.org/10.1016/j.nut.2015.11.001

Borgeraas, H., Johnson, L.K., Skattebu, J., Hertel, J.K., \& Hjelmesæth, J. (2018). Effects of probiotics on body weight, body mass index, fat mass and fat percentage in subjects with overweight or obesity: A systematic review and meta-analysis of randomized controlled trials. Obesity Reviews, 19(2), 219-232. https://doi.org/10.1111/obr.12626

Brahe, L.K., Astrup, A., \& Larsen, L.H. (2016). Can We Prevent Obesity-Related Metabolic Diseases by Dietary Modulation of the Gut Microbiota? Advances in Nutrition, 7(1), 90-101. https://doi.org/10.3945/an.115.010587

Castaner, O., Goday, A., Park, Y.M., Lee, S.H., Magkos, F., Shiow, S.A.T.E., \& Schröder, H. (2018). The Gut Microbiome Profile in Obesity: A Systematic Review. International Journal of Endocrinology, 2018. https://doi.org/10.1155/2018/4095789

Cerdó, T., García-Santos, J.A., Bermúdez, M., \& Campoy, C. (2019). The Role of Probiotics and Prebiotics in the Prevention and Treatment of Obesity. Nutrients, 11(3). https://doi.org/10.3390/nu11030635

Chung, H.J., Yu, J.G., Lee, I.A., Liu, M.J., Shen, Y.F., Sharma, S.P., Jamal, M.A.H.M., Yoo, J.H., Kim, H.J., \& Hong, S.T. (2016). Intestinal removal of free fatty acids from hosts by Lactobacilli for the treatment of obesity. FEBS Open Bio, 6(1), 6476. https://doi.org/10.1002/2211-5463.12024

Crovesy, L., Masterson, D., \& Rosado, E.L. (2020). Profile of the gut microbiota of adults with obesity: A systematic review. European Journal of Clinical Nutrition. https://doi.org/10.1038/s41430-020-0607-6

Culpepper, T., Rowe, C.C., Rusch, C.T., Burns, A.M., Federico, A.P., Girard, S.A., Tompkins, T.A., Nieves, C., Dennis-Wall, J.C., Christman, M.C., \& LangkampHenken, B. (2019). Three probiotic strains exert different effects on plasma bile acid profiles in healthy obese adults: Randomised, double-blind placebocontrolled crossover study. Beneficial Microbes, 10(5), 497-509. https://doi.org/10.3920/BM2018.0151

Dahiya, D.K., Renuka, Puniya, M., Shandilya, U.K., Dhewa, T., Kumar, N., Kumar, S., Puniya, A.K., \& Shukla, P. (2017). Gut Microbiota Modulation and Its Relationship with Obesity Using Prebiotic Fibers and Probiotics: A Review. Frontiers in Microbiology, 8. https://doi.org/10.3389/fmicb.2017.00563

de Groot, P.F., Frissen, M.N., de Clercq, N.C., \& Nieuwdorp, M. (2017). Fecal microbiota transplantation in metabolic syndrome: History, present and future. Gut Microbes, 8(3), 253-267. https://doi.org/10.1080/19490976.2017.1293224

Depommier, C., Everard, A., Druart, C., Plovier, H., Van Hul, M., Vieira-Silva, S., Falony, G., Raes, J., Maiter, D., Delzenne, N.M., de Barsy, M., Loumaye, A., Hermans, M.P., Thissen, J.P., de Vos, W.M., \& Cani, P.D. (2019). 
Supplementation with Akkermansia muciniphila in overweight and obese human volunteers: A proof-of-concept exploratory study. Nature Medicine, 25(7), 10961103. https://doi.org/10.1038/s41591-019-0495-2

Ejtahed, H.S., Angoorani, P., Soroush, A.R., Atlasi, R., Hasani-Ranjbar, S., Mortazavian, A.M., \& Larijani, B. (2019). Probiotics supplementation for the obesity management; A systematic review of animal studies and clinical trials. Journal of Functional Foods, 52, 228-242. https://doi.org/10.1016/j.jff.2018.10.039

Falalyeyeva, T.M., Leschenko, I.V, Beregova, T.V., Lazarenko, L.M., Savchuk, O.M., Sichel, L.M., Tsyryuk, O.I., Vovk, T.B., \& Spivak, M.Y. (2017). Probiotic strains of lactobacilli and bifidobacteria alter pro- and anti-inflammatory cytokines production in rats with monosodium glutamate-induced obesity. Fiziolohichnyi zhurnal, 63(1), 17-25. https://doi.org/10.15407/fz63.01.017

Fayfman, M., Flint, K., \& Srinivasan, S. (2019). Obesity, Motility, Diet, and Intestinal Microbiota-Connecting the Dots. Current Gastroenterology Reports, 21(4), 15. https://doi.org/10.1007/s11894-019-0680-y

Ferrarese, R., Ceresola, E.R., Preti, A., \& Canducci, F. (2018). Probiotics, prebiotics and synbiotics for weight loss and metabolic syndrome in the microbiome era. European Review for Medical and Pharmacological Sciences, 22(21), 7588-7605. https://doi.org/10.26355/eurrev_201811_16301

Flint, H.J., Scott, K.P., Duncan, S.H., Louis, P., \& Forano, E. (2012). Microbial degradation of complex carbohydrates in the gut. En Gut Microbes (Vol. 3, Número 4, p. 289). Taylor \& Francis. https://doi.org/10.4161/gmic.19897

Gérard, P. (2016). Gut microbiota and obesity. Cellular and molecular life sciences: CMLS, 73(1), 147-162. https://doi.org/10.1007/s00018-015-2061-5

Gomes, A.C., Hoffmann, C., \& Mota, J.F. (2018). The human gut microbiota: Metabolism and perspective in obesity. Gut Microbes, 9(4), 308-325. https://doi.org/10.1080/19490976.2018.1465157

Hadi, A., Alizadeh, K., Hajianfar, H., Mohammadi, H., \& Miraghajani, M. (2018). Efficacy of synbiotic supplementation in obesity treatment: A systematic review and meta-analysis of clinical trials. Critical Reviews in Food Science and Nutrition, O(0), 1-13. https://doi.org/10.1080/10408398.2018.1545218

Hampl, S., \& Campbell, A. (2015). Recognizing obesity and its complications: The story of Score 1 for Health. NASN School Nurse, 30(1), 46-52. https://doi.org/10.1177/1942602X14559749

Heiss, C.N., \& Olofsson, L.E. (2019). The role of the gut microbiota in development, function and disorders of the central nervous system and the enteric nervous system. Journal of Neuroendocrinology, 31(5), e12684. https://doi.org/10.1111/jne.12684 
Hibberd, A.A., Yde, C.C., Ziegler, M.L., Honoré, A.H., Saarinen, M.T., Lahtinen, S., Stahl, B., Jensen, H.M., \& Stenman, L.K. (2019). Probiotic or synbiotic alters the gut microbiota and metabolism in a randomised controlled trial of weight management in overweight adults. Beneficial Microbes, 10(2), 121-135. https://doi.org/10.3920/BM2018.0028

Higashikawa, F., Noda, M., Awaya, T., Danshiitsoodol, N., Matoba, Y., Kumagai, T., \& Sugiyama, M. (2016). Antiobesity effect of Pediococcus pentosaceus LP28 on overweight subjects: A randomized, double-blind, placebo-controlled clinical trial. European Journal of Clinical Nutrition, 70(5), 582-587. https://doi.org/10.1038/ejcn.2016.17

Huang, C.H., Ho, C.Y., Chen, C.T., Hsu, H.F., \& Lin, Y.H. (2019). Probiotic BSH activity and anti-obesity potential of lactobacillus plantarum strain TCI378 isolated from Korean Kimchi. Preventive Nutrition and Food Science, 24(4), 434441. https://doi.org/10.3746/pnf.2019.24.4.434

Hulston, C.J., Churnside, A.A., \& Venables, M.C. (2015). Probiotic supplementation prevents high-fat, overfeeding-induced insulin resistance in human subjects. British Journal of Nutrition, 113(4), 596-602. https://doi.org/10.1017/S0007114514004097

Ianiro, G., Tilg, H., \& Gasbarrini, A. (2016). Antibiotics as deep modulators of gut microbiota: Between good and evil. Gut, 65(11), 1906-1915. https://doi.org/10.1136/gutjnl-2016-312297

Ivey, K.L., Hodgson, J.M., Kerr, D.A., Lewis, J.R., Thompson, P.L., \& Prince, R.L. (2014). The effects of probiotic bacteria on glycaemic control in overweight men and women: A randomised controlled trial. European journal of clinical nutrition, 68(4), 447-452. https://doi.org/10.1038/ejcn.2013.294

Jadad, A.R., Moore, R.A., Carroll, D., Jenkinson, C., Reynolds, D.J., Gavaghan, D.J., \& McQuay, H.J. (1996). Assessing the quality of reports of randomized clinical trials: Is blinding necessary? Controlled Clinical Trials, 17(1), 1-12. https://doi.org/10.1016/0197-2456(95)00134-4

Jung, S.P., Lee, K.M., Kang, J.H., Yun, I.S., Park, H.O., Moon, Y., \& Kim, J.Y. (2013). Effect of Lactobacillus gasseri BNR17 on overweight and obese adults: A randomized, double-blind clinical trial. Korean Journal of Family Medicine, 34(2), 80-89. https://doi.org/10.4082/kjfm.2013.34.2.80

Kadooka, Y., Sato, M., Imaizumi, K., Ogawa, A., Ikuyama, K., Akai, Y., Okano, M., Kagoshima, M., \& Tsuchida, T. (2010). Regulation of abdominal adiposity by probiotics (Lactobacillus gasseri SBT2055) in adults with obese tendencies in a randomized controlled trial. European Journal of Clinical Nutrition, 64(6), 636643. https://doi.org/10.1038/ejcn.2010.19

Kadooka, Y., Sato, M., Ogawa, A., Miyoshi, M., Uenishi, H., Ogawa, H., Ikuyama, K., Kagoshima, M., \& Tsuchida, T. (2013). Effect of Lactobacillus gasseri SBT2055 in fermented milk on abdominal adiposity in adults in a randomised controlled 
Probiotics in obesity and overweight

trial. British Journal of Nutrition, 110(09), 1696-1703. https://doi.org/10.1017/S0007114513001037

Karl, J.P., Margolis, L.M., Madslien, E.H., Murphy, N.E., Castellani, J.W., Gundersen, Y., Hoke, A.V., Levangie, M.W., Kumar, R., Chakraborty, N., Gautam, A., Hammamieh, R., Martini, S., Montain, S.J., \& Pasiakos, S.M. (2017). Changes in intestinal microbiota composition and metabolism coincide with increased intestinal permeability in young adults under prolonged physiological stress. American Journal of Physiology. Gastrointestinal and Liver Physiology, 312(6), G559-G571. https://doi.org/10.1152/ajpgi.00066.2017

Kim, J., Yun, J.M., Kim, M.K., Kwon, O., \& Cho, B. (2018). Lactobacillus gasseri BNR17 Supplementation Reduces the Visceral Fat Accumulation and Waist Circumference in Obese Adults: A Randomized, Double-Blind, PlaceboControlled Trial. Journal of Medicinal Food, 21(5), 454-461. https://doi.org/10.1089/jmf.2017.3937

King, R.J., \& Ajjan, R.A. (2016). Vascular risk in obesity: Facts, misconceptions and the unknown. Diabetes and Vascular Disease Research, 14(1), 2-13. https://doi.org/10.1177/1479164116675488

Koutnikova, H., Genser, B., Monteiro-Sepulveda, M., Faurie, J.M., Rizkalla, S., Schrezenmeir, J., \& Clément, K. (2019). Impact of bacterial probiotics on obesity, diabetes and non-alcoholic fatty liver disease related variables: A systematic review and meta-analysis of randomised controlled trials. BMJ Open, 9(3), e017995. https://doi.org/10.1136/bmjopen-2017-017995

Leber, B., Tripolt, N. J., Blattl, D., Eder, M., Wascher, T. C., Pieber, T. R., Stauber, R., Sourij, H., Oettl, K., \& Stadlbauer, V. (2012). The influence of probiotic supplementation on gut permeability in patients with metabolic syndrome: An open label, randomized pilot study. European Journal of Clinical Nutrition, 66(10), 1110-1115. https://doi.org/10.1038/ejen.2012.103

Lee, S.J., Bose, S., Seo, J.G., Chung, W.S., Lim, C.Y., \& Kim, H. (2014). The effects of co-administration of probiotics with herbal medicine on obesity, metabolic endotoxemia and dysbiosis: A randomized double-blind controlled clinical trial. Clinical Nutrition, 33(6), 973-981. https://doi.org/10.1016/j.clnu.2013.12.006

Li, J., Lin, S., Vanhoutte, P.M., Woo, C. W., \& Xu, A. (2016). Akkermansia Muciniphila Protects Against Atherosclerosis by Preventing Metabolic Endotoxemia-Induced Inflammation in Apoe-/- Mice. Circulation, 133(24), 2434-2446. https://doi.org/10.1161/CIRCULATIONAHA.115.019645

López-Moreno, A., Suárez, A., Avanzi, C., Monteoliva-Sánchez, M., \& Aguilera, M. (2020). Probiotic Strains and Intervention Total Doses for Modulating ObesityRelated Microbiota Dysbiosis: A Systematic Review and Meta-analysis. Nutrients, 12(7). https://doi.org/10.3390/nu12071921

Madjd, A., Taylor, M.A., Mousavi, N., Delavari, A., Malekzadeh, R., Macdonald, I.A., \& Farshchi, H.R. (2016). Comparison of the effect of daily consumption of 
probiotic compared with low-fat conventional yogurt on weight loss in healthy obese women following an energy-restricted diet: A randomized controlled trial. American Journal of Clinical Nutrition, 103(2), 323-329. https://doi.org/10.3945/ajcn.115.120170

Maldonado-Galdeano, C., Cazorla, S.I., Lemme-Dumit, J.M., Vélez, E., \& Perdigón, G. (2019). Beneficial Effects of Probiotic Consumption on the Immune System. Annals of Nutrition and Metabolism, 74(2), 115-124. https://doi.org/10.1159/000496426

Maruvada, P., Leone, V., Kaplan, L.M., \& Chang, E.B. (2017). The human microbiome and obesity: Moving beyond associations. Cell Host \& Microbe, 22(5), 589-599. https://doi.org/10.1016/j.chom.2017.10.005

Mitev, K., \& Taleski, V. (2019). Association between the Gut Microbiota and Obesity. Open Access Macedonian Journal of Medical Sciences, 7(12), 2050-2056. https://doi.org/10.3889/oamjms.2019.586

Moher, D., Liberati, A., Tetzlaff, J., Altman, D.G., \& Altman, D. (2009). Preferred Reporting Items for Systematic Reviews and Meta-Analyses: The PRISMA Statement. PLoS Medicine, 6(7), e1000097. https://doi.org/10.1371/journal.pmed.1000097

Nakamura, F., Ishida, Y., Aihara, K., Sawada, D., Ashida, N., Sugawara, T., Aoki, Y., Takehara, I., Takano, K., \& Fujiwara, S. (2016). Effect of fragmented Lactobacillus amylovorus CP1563 on lipid metabolism in overweight and mildly obese individuals: A randomized controlled trial. Microbial Ecology in Health \& Disease, 27(0). https://doi.org/10.3402/mehd.v27.30312

Noh, K., Kang, Y.R., Nepal, M.R., Shakya, R., Kang, M.J., Kang, W., Lee, S., Jeong, H.G., \& Jeong, T.C. (2017). Impact of gut microbiota on drug metabolism: An update for safe and effective use of drugs. Archives of Pharmacal Research, 40(12), 1345-1355. https://doi.org/10.1007/s12272-017-0986-y

Novotny-Núñez, I., Maldonado-Galdeano, C., de Moreno de LeBlanc, A., \& Perdigón, G. (2015). Lactobacillus casei CRL 431 administration decreases inflammatory cytokines in a diet-induced obese mouse model. Nutrition, 31(7-8), 1000-1007. https://doi.org/10.1016/j.nut.2015.02.006

Obata, Y., \& Pachnis, V. (2016). The Effect of Microbiota and the Immune System on the Development and Organization of the Enteric Nervous System. Gastroenterology, 151(5), 836-844. https://doi.org/10.1053/j.gastro.2016.07.044

Ogawa, A., Kadooka, Y., Kato, K., Shirouchi, B., \& Sato, M. (2014). Lactobacillus gasseri SBT2055 reduces postprandial and fasting serum non-esterified fatty acid levels in Japanese hypertriacylglycerolemic subjects. Lipids in Health and Disease, 13(1), 36. https://doi.org/10.1186/1476-511X-13-36

Osterberg, K.L., Boutagy, N.E., McMillan, R.P., Stevens, J.R., Frisard, M.I., Kavanaugh, J.W., Davy, B.M., Davy, K.P., \& Hulver, M.W. (2015). Probiotic 
supplementation attenuates increases in body mass and fat mass during high-fat diet in healthy young adults. Obesity, 23(12), 2364-2370. https://doi.org/10.1002/oby.21230

Paramsothy, S., Paramsothy, R., Rubin, D.T., Kamm, M.A., Kaakoush, N.O., Mitchell, H.M., \& Castaño-Rodríguez, N. (2017). Faecal Microbiota Transplantation for Inflammatory Bowel Disease: A Systematic Review and Meta-analysis. Journal of Crohn's \& Colitis, 11(10), 1180-1199. https://doi.org/10.1093/ecco-jcc/jjx063

Park, S.S., Lee, Y.J., Kang, H., Yang, G., Hong, E.J., Lim, J.Y., Oh, S., \& Kim, E. (2019). Lactobacillus amylovorus KU4 ameliorates diet-induced obesity in mice by promoting adipose browning through PPAR $\gamma$ signaling. Scientific Reports, 9(1), 1-10. https://doi.org/10.1038/s41598-019-56817-w

Pedret, A., Valls, R.M., Calderón-Pérez, L., Llauradó, E., Companys, J., Pla-Pagà, L., Moragas, A., Martín-Luján, F., Ortega, Y., Giralt, M., Caimari, A., Chenoll, E., Genovés, S., Martorell, P., Codoñer, F.M., Ramón, D., Arola, L., \& Solà, R. (2019). Effects of daily consumption of the probiotic Bifidobacterium animalis subsp. Lactis CECT 8145 on anthropometric adiposity biomarkers in abdominally obese subjects: A randomized controlled trial. International Journal of Obesity, 43(9), 1863-1868. https://doi.org/10.1038/s41366-018-0220-0

Prados-Bo, A., Gómez-Martínez, S., Nova, E., \& Marcos, A. (2015). El papel de los probióticos en el manejo de la obesidad. Nutricion Hospitalaria, 31, 10-18. https://doi.org/10.3305/nh.2015.31.sup1.8702

Ramírez-Pérez, O., Cruz-Ramón, V., Chinchilla-López, P., \& Méndez-Sánchez, N. (2017). The role of the gut microbiota in bile acid metabolism. Annals of Hepatology, 16, S21-s26. https://doi.org/10.5604/01.3001.0010.5494

Rizzetto, L., Fava, F., Tuohy, K.M., \& Selmi, C. (2018). Connecting the immune system, systemic chronic inflammation and the gut microbiome: The role of sex. Journal of Autoimmunity, 92(April), 12-34. https://doi.org/10.1016/j.jaut.2018.05.008

Rouxinol-Dias, A.L., Pinto, A.R., Janeiro, C., Rodrigues, D., Moreira, M., Dias, J., \& Pereira, P. (2016). Probiotics for the control of obesity - Its effect on weight change. Porto Biomedical Journal, 1(1), 12-24. https://doi.org/10.1016/j.pbj.2016.03.005

Sanchis-Chordà, J., del Pulgar, E.M.G., Carrasco-Luna, J., Benítez-Páez, A., Sanz, Y., \& Codoñer-Franch, P. (2018). Bifidobacterium pseudocatenulatum CECT 7765 supplementation improves inflammatory status in insulin-resistant obese children. European Journal of Nutrition, O(0), 0. https://doi.org/10.1007/s00394-018-18285

Sarao, L.K., \& Arora, M. (2017). Probiotics, prebiotics, and microencapsulation: A review. Critical Reviews in Food Science and Nutrition, 57(2), 344-371. https://doi.org/10.1080/10408398.2014.887055 
Stenman, L.K., Lehtinen, M.J., Meland, N., Christensen, J.E., Yeung, N., Saarinen, M. T., Courtney, M., Burcelin, R., Lähdeaho, M.L., Linros, J., Apter, D., Scheinin, M., Kloster-Smerud, H., Rissanen, A., \& Lahtinen, S. (2016). Probiotic With or Without Fiber Controls Body Fat Mass, Associated With Serum Zonulin, in Overweight and Obese Adults-Randomized Controlled Trial. EBioMedicine, 13, 190-200. https://doi.org/10.1016/j.ebiom.2016.10.036

Stone, P.W. (2002). Popping the (PICO) question in research and evidence-based practice. Applied nursing research: ANR, 15(3), 197-198. https://doi.org/10.1053/apnr.2002.34181

Sun, Q., Zhang, S., Liu, X., Huo, Y., Su, B., \& Li, X. (2020). Effects of a probiotic intervention on Escherichia coli and high-fat diet-induced intestinal microbiota imbalance. Applied Microbiology and Biotechnology, 104(3), 1243-1257. https://doi.org/10.1007/s00253-019-10304-4

Suzumura, E.A., Bersch-Ferreira, Â.C., Torreglosa, C.R., da Silva, J.T., Coqueiro, A.Y., Kuntz, M.G.F., Chrispim, P.P., Weber, B., \& Cavalcanti, A.B. (2019). Effects of oral supplementation with probiotics or synbiotics in overweight and obese adults: A systematic review and meta-analyses of randomized trials. Nutrition Reviews, 77(6), 430-450. https://doi.org/10.1093/nutrit/nuz001

Takahashi, S., Anzawa, D., Takami, K., Ishizuka, A., \& Mawatari, T. (2016). On visceral fat accumulation in healthy Japanese adults: A randomized controlled trial. Bioscience of Microbiota, Food and Healt, 35(4), 163-171.

The Food and Agriculture Organization of the United Nations/World Health Organization (FAO/WHO) (2001). Health and Nutritional Properties of Probiotics in Food including Powder Milk with Live Lactic Acid Bacteria. http://www.fao.org/tempref/docrep/fao/meeting/009/y6398e.pdf

Turnbaugh, P.J., Ley, R.E., Mahowald, M.A., Magrini, V., Mardis, E.R., \& Gordon, J.I. (2006). An obesity-associated gut microbiome with increased capacity for energy harvest. Nature, 444(7122), 1027-1031. https://doi.org/10.1038/nature05414

Vallianou, N., Stratigou, T., Christodoulatos, G.S., Tsigalou, C., \& Dalamaga, M. (2020). Probiotics, Prebiotics, Synbiotics, Postbiotics, and Obesity: Current Evidence, Controversies, and Perspectives. Current Obesity Reports. https://doi.org/10.1007/s13679-020-00379-w

Wang, B.Z., Xin, S.S., Ding, L.N., Ding, W.Y., Hou, Y.L., Liu, C.Q., \& Zhang, X.D. (2019). The Potential Role of Probiotics in Controlling Overweight/Obesity and Associated Metabolic Parameters in Adults: A Systematic Review and MetaAnalysis. Evidence-based Complementary and Alternative Medicine, 2019. https://doi.org/10.1155/2019/3862971

World Health Organization (WHO) (2018). World Health Statistics 2018: Monitoring health for the SDGs. World Health Organization. https://apps.who.int/iris/bitstream/handle/10665/272596/9789241565585-

eng.pdf 
Xu, Y., Wang, N., Tan, H.Y., Li, S., Zhang, C., \& Feng, Y. (2020). Function of Akkermansia muciniphila in Obesity: Interactions With Lipid Metabolism, Immune Response and Gut Systems. Frontiers in Microbiology, 11. https://doi.org/10.3389/fmicb.2020.00219

Zarrati, M., Salehi, E., Mofid, V., Hossein Zadeh-Attar, M.J., Nourijelyani, K., Bidad, K., \& Shidfar, F. (2013). Relationship between probiotic consumption and IL-10 and IL-17 secreted by PBMCs in overweight and obese people. Iranian journal of allergy, asthma, and immunology, 12(4), 404-406.

Zarrati, M., Salehi, E., Nourijelyani, K., Mofid, V., Zadeh, M.J.H., Najafi, F., Ghaflati, Z., Bidad, K., Chamari, M., Karimi, M., \& Shidfar, F. (2014). Effects of probiotic yogurt on fat distribution and gene expression of proinflammatory factors in peripheral blood mononuclear cells in overweight and obese people with or without weight-loss diet. Journal of the American College of Nutrition, 33(6), 417-425. https://doi.org/10.1080/07315724.2013.874937

Zhai, S., Qin, S., Li, L., Zhu, L., Zou, Z., \& Wang, L. (2019). Dietary butyrate suppresses inflammation through modulating gut microbiota in high-fat diet-fed mice. FEMS microbiology letters, 366(13), fnz153. https://doi.org/10.1093/femsle/fnz153 